\title{
Design, Execution and Rebuilding of a Plasma Wind Tunnel Test Compared with an Advanced Infrared Measurement Technique
}

\author{
Marco Di Clemente, Giuseppe Rufolo, \\ Francesco Battista and Adolfo Martucci \\ Italian Aerospace Research Centre \\ Italy
}

\section{Introduction}

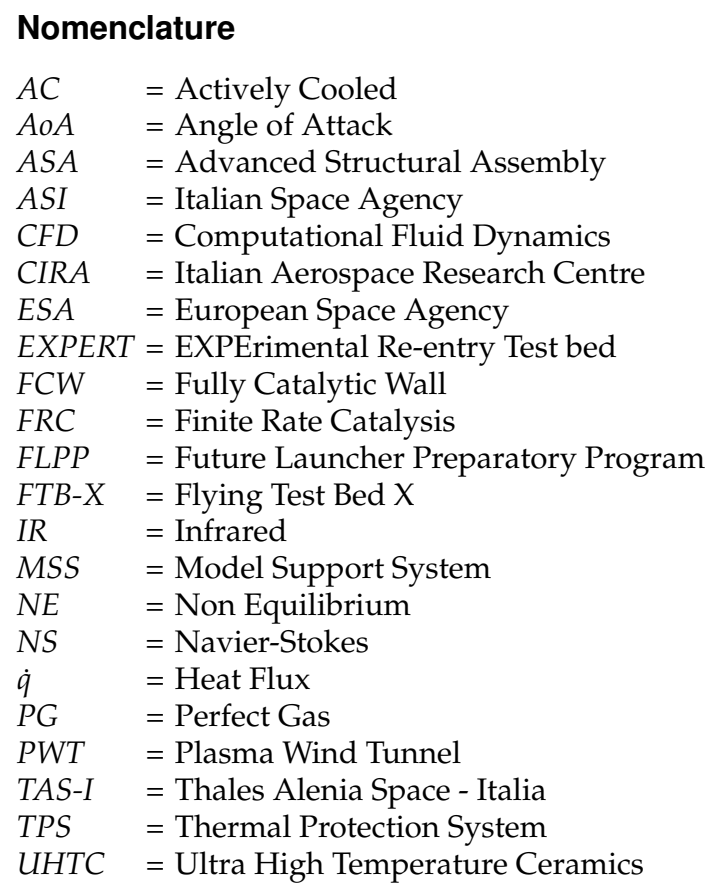

The extreme difficulties of testing, in a flight environment, technologies developed for the thermal protection of a re-entry vehicle put emphasis on the validation of numerical prediction tools. The ground testing in a Plasma Wind Tunnel facility entails a series of limitations in terms of cost and representativeness of the flight environment; therefore to found the way of improving CFD tools, both with flight and ground experimental data, is the key for a more reliable and robust Thermal Protection System (TPS) design. Existing in-flight measurements database are extremely poor and the need for improving them is testified by actual European program as EXPERT (Ratti et al., 2008) or FLPP-IXV (Tumino, 
2006). On a parallel way it is also fundamental to improve the reliability of the experimental data acquired from ground tests. The validation of numerical methodology with ground measurements necessarily asks for a correct rebuilding of the test. To this aim, in the frame of the ASA program, a technological program carried out in Italy in the past years, funded by the Italian Space Agency, different TPS technologies have been developed and then tested under representative conditions not only to validate the design tools but also to gather data to be used for code validation. ASA program faced the aerothermal heating on a wing leading edge of a re-entry vehicle by developing, four TPS technologies for the different parts of the wing, namely two interchangeable systems for the leading edge (an UHTC-based and an actively cooled leading edge) and two for the panels (an Hybrid C/C and a Metal Matrix Composite panel); the experimental vehicle FTB-X, whose preliminary analysis was carrying out in the framework of the USV program (Pezzella et al., 2007), was considered as reference target in terms of thermal loads to be handled by the thermal protection system. The project team, leaded by TAS-I and with the cooperation of different italian research centres and institutions, encompassed the development of these technologies and their qualification during different tests performed in the Plasma Wind Tunnel Scirocco. In the present analysis, the definition of the requirements, derived from the analysis of the FTB-X trajectory, the design and rebuilding of one of the performed tests, will be presented in order to validate an aerothermal coupling procedure developed. Traditionally, an aerodynamicist assumes a rigid isothermal or adiabatic body, with or without radiative equilibrium assumption, in order to predict surface pressure and heating rate. The aerodynamic heating is used to compute the temperature distribution inside the structure by means of a heat transfer analysis. Such an uncoupled approach may result to be quite inaccurate especially in a case, as the present one, in which the test procedure foresee a variation of flow condition and model attitude and the material to be tested has a relatively high thermal conductivity. Therefore, an integrated procedure to couple the external aerodynamic field to the internal thermal state of the structure has been adopted for the numerical rebuilding. The results of such aerothermal rebuilding have been compared with the experimental data provided by an Advanced Infrared Thermo-camera technique.

\section{Model description: geometry and materials}

The main purpose of the Advanced Structural Assembly project was to qualify, in an high enthalpy ground facility, a certain number of new technologies potentially applicable as wing thermal protection system to new generation of re-entry vehicles; to this aim it was proposed to realize an adequate test article to be tested in Scirocco, the CIRA Plasma Wind Tunnel (PWT) facility (De Filippis et al., 2003), that should be representative of the wing of FTB-X vehicle. The test article has been conceived to be compatible with the facility itself in terms of dimensions, sustainable weights, auxiliary requested equipments, available measurement systems, etc., by guaranteeing the most valuable scientific feedback and, at the same time, an adequate safety level. As a matter of fact, it cannot be possible to test a real full-scale delta wing complete of the fuselage in the existing plasma facilities. The presence of chemical effects does not allow to simply scale the geometry to wind tunnels allowable dimensions; moreover, in the present case, the need to have a full scale test article is due to the necessity to test TPS technologies developed for flight. Moreover, it makes no sense to test only a portion of the delta wing because of the non-reproducibility of the real three-dimensional effects. For this reason it was decided to realize the test article by extruding a longitudinal section directly derived from FTB- $X$ wing as described in Fig. 1. 


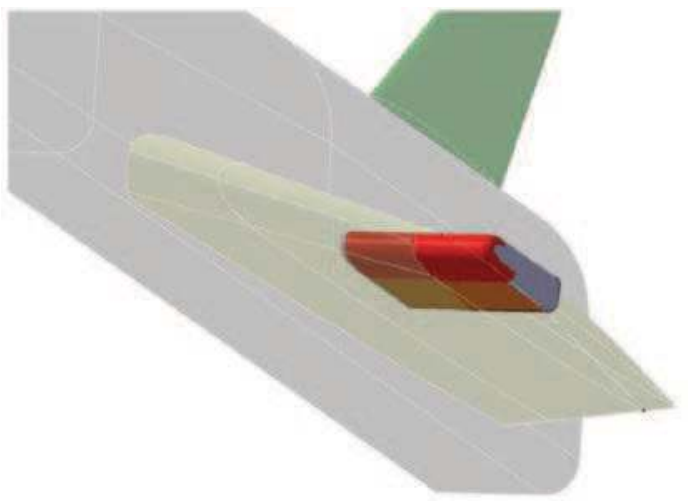

Fig. 1. FTB-X wing and test article derivation

Two lateral rounded fairings have been also defined in order to reduce as much as possible the overheating due to the finite span effects of the test article. Additionally some small modifications to the original wing section profile were necessary in order both to simplify the assembly and to allow the compatibility of the test article with the PWT. The conceived test article has been used to test in the PWT facility the innovative thermal protection system technologies and materials suitable for re-entry vehicles and thermo-structural applications, developed in the frame of the same project. In detail, four different technologies were developed and tested within the ASA project, applicable to the different parts of a wing (two for the leading edge and two, respectively, for the windward and leeward side) but, for what concerns the aims of the present work, only the so-called FTB-2 configuration will be taken under consideration. In this case, the test article is equipped with an actively cooled leading edge, a Metal Matrix panel on the windward (which is the object of the present analysis) and an Hybrid C/C panel on the leeward side. As a matter of fact from the test design activity, that will be described hereinafter, it comes out that in order to reproduce as much as possible the in-flight heat flux distribution of the FTB-X delta wing over the designed test article with no-sweep angle it is necessary to perform the test at an angle of attack of at least 35 deg while the angle of attack along the flight trajectory was $25 \mathrm{deg}$ at its maximum. To this aim it was decided to have a mechanical incidence of $25 \mathrm{deg}$ provided by the model holder and to manage the remaining deflection angle by means of the moveble facility Model Support System (MSS). In Fig.2 the test article mounted on the MSS and ready to be tested is shown.

\section{Numerical aspects}

In the following sections the numerical results obtained for the rebuilding of the test will be presented; hereinafter, some details regarding the numerical codes used for the computations and the grids for the spatial discretization are presented.

\subsection{Numerical codes CFD code}

CIRA code H3NS has been used to perform the external flowfield computations. It is a structured multiblock finite volume solver that allow the treatment of a wide range of compressible fluid dynamics problems. The fluid can be treated or as a prefect gas or as a mixture of perfect gases in the case of thermo-chemical non equilibrium flows. In the latter case the chemical model for air is due to Park and it is characterized by 17 reactions between 


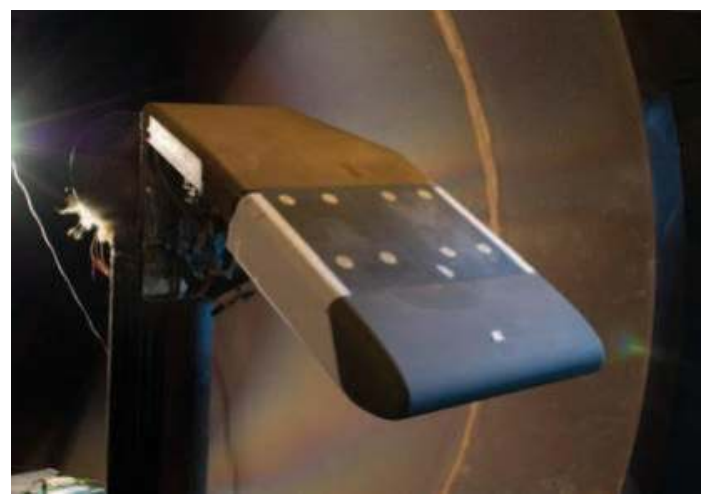

Fig. 2. Test Article with the model holder and the PWT Model Support System

the five species $\left(\mathrm{O}, \mathrm{N}, \mathrm{NO}, \mathrm{O}_{2}, \mathrm{~N}_{2}\right)$, neglecting the presence of noble gas in the air (e.g. Ar). The energy exchange between vibrational and translational modes (TV) is modelled with the classical Landau-Teller non-equilibrium equation, with average relaxation times taken from the Millikan-White theory modified by Park. For what concerns transport coefficient, the viscosity of the single species is evaluated by a fit of collision integrals calculated by Yun and Mason, the thermal conductivity is calculated by means of the Eucken law; the viscosity and thermal conductivity of the gas mixture are then calculated by using the semi-empirical Wilke formulas. The diffusion of the multi-component gas is computed through a sum rule of the binary diffusivities of each couple of species (from the tabulated collision integrals of Yun and Mason). Transport coefficient, in the hypothesis of an ideal gas, are derived from Sutherland law, suitably modified to take into account low temperature conditions. With respect to the numerical formulation, conservation equations, in integral form, are discretized with a finite volume, cell centred, technique. Eulerian flux are computed with a Flux Difference Splitting method (Borrelli \& Pandolfi, 1990). Second order formulation is obtained by means of an Essentially Non Oscillatory reconstruction of interface value. Viscous flux are computed with a classical centred scheme. Integration in time is performed by employing an explicit multistage Runge-Kutta algorithm coupled with an implicit evaluation of the source terms.

\section{Thermal code}

The numerical solution of the thermal field inside the solid is carried out by means of an unsteady explicit two-dimensional multiblock in house developed code (Di Clemente et al., 2008). The code is capable of solving the heat conduction equation over a generic $2 \mathrm{D}$ or axi-symmetric geometry provided a structured quadrilateral multiblock grid. A finite volume approach is used for the spatial discretization, i.e. for each cell the laplacian term of the heat equation is treated as the divergence of the temperature gradient and the Gauss theorem is applied to transform the divergence into a surface integral over the cell border of the flux of the temperature derivative. A nine points centered stencil is adopted for the flux discretization, thus allowing an higher accuracy with respect to the classical five points stencil especially for irregular grids (high aspect ratio and/or skeweness of the cells). The time integration is based on a first-order explicit Euler integration. The code allow the use of temperature dependent material properties: thermal conductivity, heat capacity, emissivity. Different types of boundary conditions have been implemented in order to allow different kind of coupling with the external flow field:

- fixed temperature; 
- fixed heat flux;

- adiabatic wall;

- heat flux variable in time (trajectory simulation) and variable along the geometry;

- as the previous with radiation of the wall;

- heat flux varying also with temperature by using a time and space distribution of the convective coefficient.

- as the previous with radiation of the wall;

In case of aerothermal coupled simulation the transferring of information with the external flow is allowed also if the fluid and solid grid are not coincident. To this aim a suitable interpolation procedure has been developed to transfer the heat flux deriving from the flow field computation to the solid wall and viceversa to transfer the wall temperature deriving from the thermal computation to the boundary condition of the external flow solver. Concerning the accuracy in time of the results it has to be said that despite the first order of the adopted scheme, the explicit character of the solver impose very small $\delta \mathrm{t}$ thus preserving the final accuracy of the results.

\subsection{Grids for computations of the vehicle}

In this section, some details about the computational grids used for the simulation of the flow field around the vehicle, are given. As it will be clarified hereinafter, the simulation along the trajectory has been done according to a simplified methodology to reduce CPU time; for this reason, a combination of two dimensional and three dimensional computations has been adopted therefore different grids have been considered. In particular, Fig.3(a) shows the grids used for the $2 \mathrm{D}$ wing section calculations, the number of grid points is $80 \times 72$; the grids are stretched to the wall with $\Delta y_{\text {wall }}$ of $10^{-6} \mathrm{~m}$ in order to perform viscous calculations; Fig.3(b) shows the grid used to perform 3D wing-alone computations characterised by 350000 points and a $\Delta y_{\text {wall }}$ of $10^{-6} \mathrm{~m}$; in Fig.3(c) it is reported the 3D Eulerian grid and topology used for the fuselage whose points are 365000 .

\subsection{Grids for computations of the test article}

Different computational grids have been generated with the commercial grid generator IcemCFD either for the three-dimensional mesh around the entire model either for the two-dimensional simulations carried out to design the test. The topology of the mesh is characterized by 38 structured blocks with a double C-type grid around the wing model, in the longitudinal and lateral direction; moreover an O-type local topology has been used to describe the lateral extremity of the model. The block decomposition is shown in Fig. 4. Of course, due to the symmetry of both the flow and the model, only an half of the model has been simulated in order to reduce the needed CPU time. In order to verify the spatial convergency of the results, three different grid levels have been considered: each level is obtained by the previous one by doubling the number of cells in each directions. The total number of the cells for the finest level, which assures spatially converged results, is about 1.7 millions.

In Fig. 5(a) the grid on the symmetry plane, used also for the two-dimensional simulations, is shown; a local grid refinement in the region of the bow shock in front of the model has been done in order to better describe the steep gradient of flow variables and to reduce the numerical instabilities which can arise in this area. In Fig. 5(b) it is shown the detail of the wing tip. 


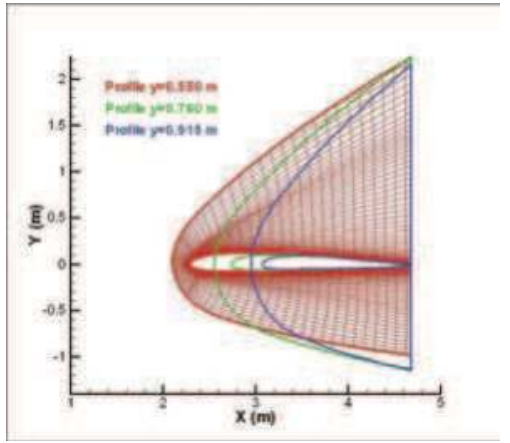

(a) Grid for 2D computations

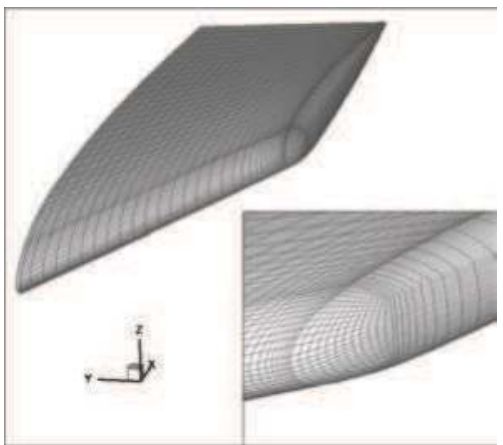

(b) Grid for wing computations

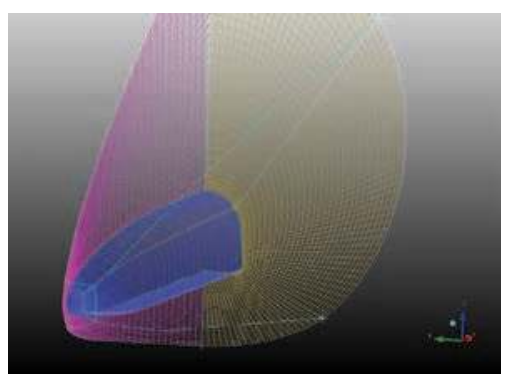

(c) Grid for 3D computations

Fig. 3. Computational grids

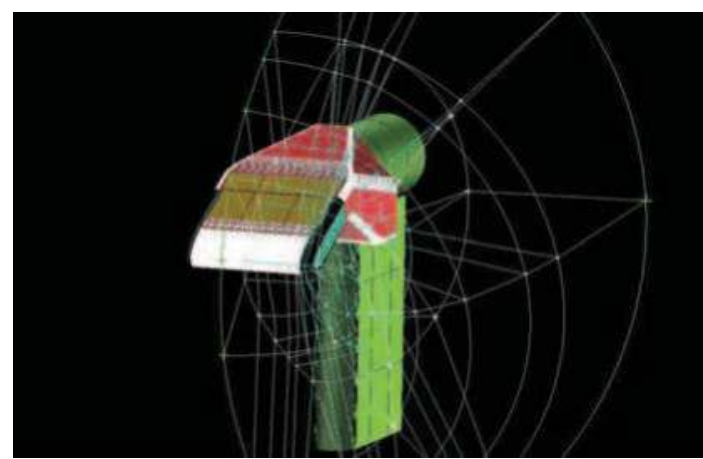

Fig. 4. Block decomposition around the test article

\subsection{Grid for internal field}

As it will be clarified hereinafter, the numerical rebuilding of the PWT test has been done through an aero-thermal coupled methodology which foreseen the computation of either the external flowfield surrounding the model either the internal thermal field inside the model. In particular, the attention of the rebuilding has been focused on the MMC panel in the upper part of the model. Thermal computations inside the panel have been carried out considering 


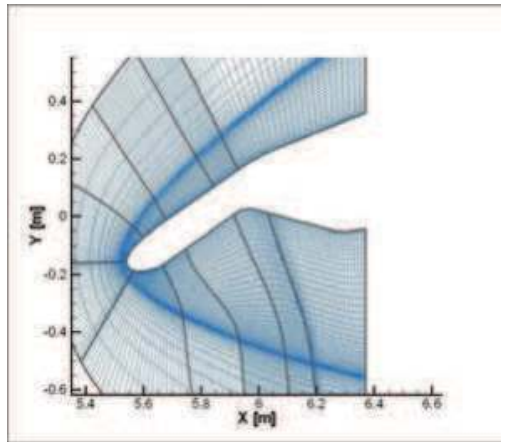

(a) plane of simmetry

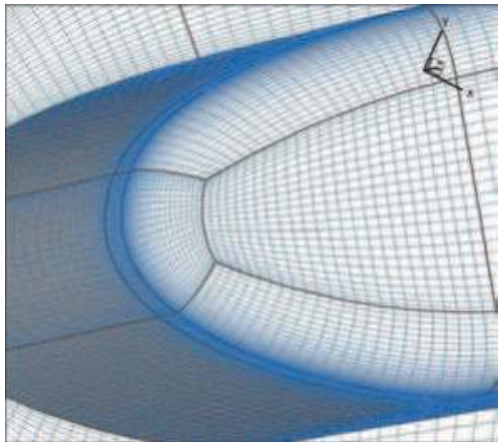

(b) detail of the tip

Fig. 5. Computational mesh

a two-dimensional section neglecting the heat conduction in the lateral direction. The grid used for these computations is shown in Fig. 6; points distribution along the wall is the same of the distribution used for the CFD computations, in order to simplify the exchange of informations (heat flux and temperature) between the external and the internal fields. In the direction normal to the wall it has been suitably tuned to describe in detail the region of stronger thermal gradients. The panel is formed by a metal matrix of $2 \mathrm{~mm}$ thickness and an insulator in the lower part of $2.5 \mathrm{~cm}$ thickness which have been discretized with two different blocks (see the green and blue mesh in Fig.6(b)).

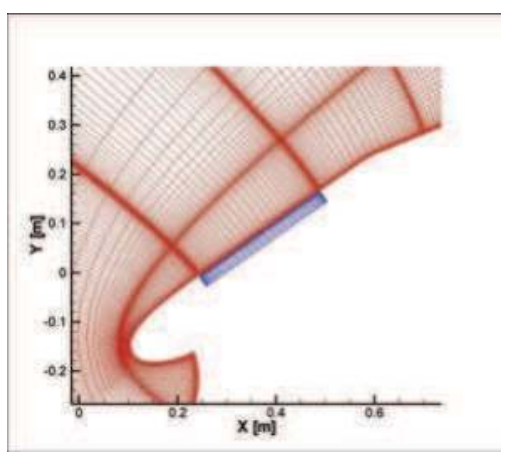

(a) plane of simmetry

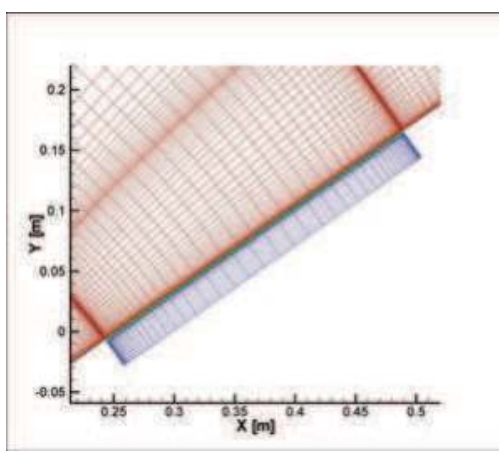

(b) detail of the panel

Fig. 6. Computational mesh for thermal computation

\section{Definition of requirements}

The development of the wing leading edge thermal protection system for a re-entry vehicle requires a deep understanding of the aero-thermal environment surrounding the vehicle; among the others the two key parameters that influence the selection and the design of a TPS suitable for a certain vehicle and trajectory are the peak-heating rate and the integrated heating over the time along the flight. The former determines the maximum temperature environment, and thus the materials selection, whereas the latter determines the thermal 
budget that the structure has to manage either with a passive insulation material and/or with an active cooling system. Due to the lack of experimental data concerning the thermal database of the FTB-X vehicle, and the impossibility to carry out an accurate numerical investigation of the aero-heating phenomenology, which would involve an high number of full three dimensional thermo-chemical non equilibrium Navier-Stokes simulations of the vehicle configuration along the flight path, a scaling simplified methodology to evaluate the time history of the aerothermal environment over the wing, capable of giving results as much reliable as possible in a reasonable time available, have been developed and applied (for major details, see Battista et al. (2007)); the level of approximation of this approach has been considered suitable for the purposes of the overall project whose main aim was to develop technologies for a re-entry vehicle under representative loads and not to design the real TPS system of the FTB-X vehicle. In the simplified methodology a series of two dimensional planar computations along the trajectory has been performed on different profiles of the wing, shown in Fig. 7, to describe the time evolution of the aerothermal environment and, in order to take into account the realistic three dimensional features of the investigated flow, over the finite span length and swept wing, the two dimensional results have been suitably scaled by means of $3 \mathrm{D}$ computations carried out over a reduced number of trajectory points.

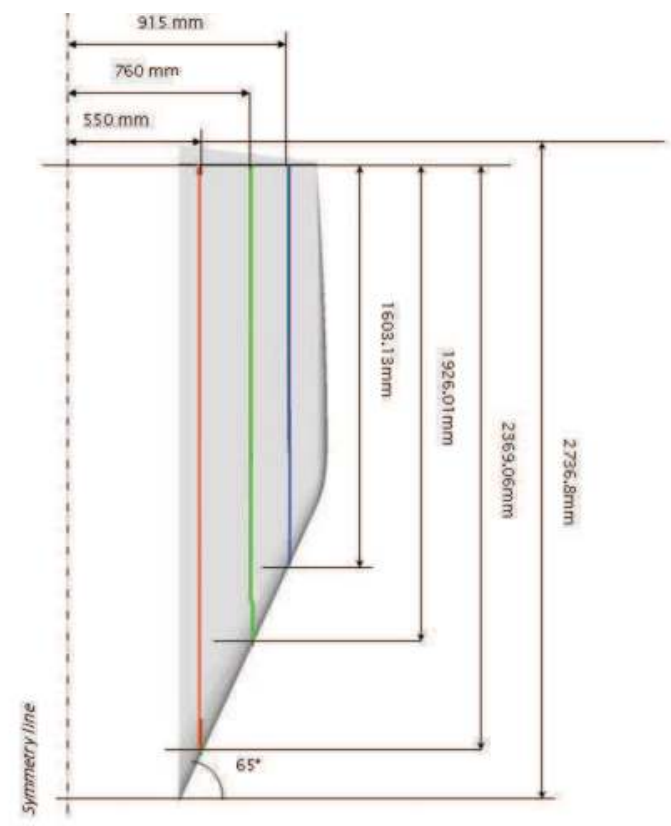

Fig. 7. Wing planform

A preliminary assessment analysis has been done to find out the most reliable conditions to perform the two dimensional non equilibrium calculations. In particular, it has been analyzed the boundary layer state (laminar/turbulent) and the wall boundary conditions in terms of catalysis and wall temperature. In this simplified methodology for the heat flux estimation over the wing no attempt has been made to describe regions of interaction of the fuselage bow shock with the leading edge bow shock. Even though this choice allowed for a relatively easy and reliable (except for the profiles interested by the shock-shock interaction) evaluation of the 
heat flux over the wing along the flight trajectory, the problem of the shock-shock interaction has been analyzed during a refinement of the present analsyis since this type of interaction can cause highly localized peaks in the heat flux distribution (Edney, 1968).

Referring to the flowchart shown in Fig. 8, the scaling methodology consists mainly of two different steps:

- determining the most reliable flow conditions ahead each wing profile: as matter of fact along the trajectory, a part of the wing is positioned inside the bow shock coming from the nose; therefore the wing sections that falls inside the fuse bow shock are exposed to a flow field with different characteristics with respect to the freestream conditions and this is accounted for considering three-dimensional computations around the fuselage and by evaluating from these computations the freestream conditions to be used for the computations around the selected wing profiles;

- two dimensional computations are performed, in non-equilibrium conditions, on the selected wing profiles along the trajectory considering the freestream conditions determined at the previous point;

- scaling of two-dimensional results to take into account the sweep angle and three dimensional effects, mainly induced by the finite length of the wing; this is done by comparing the two-dimensional and three-dimensional wing alone computations performed in some points of the flight trajectory.

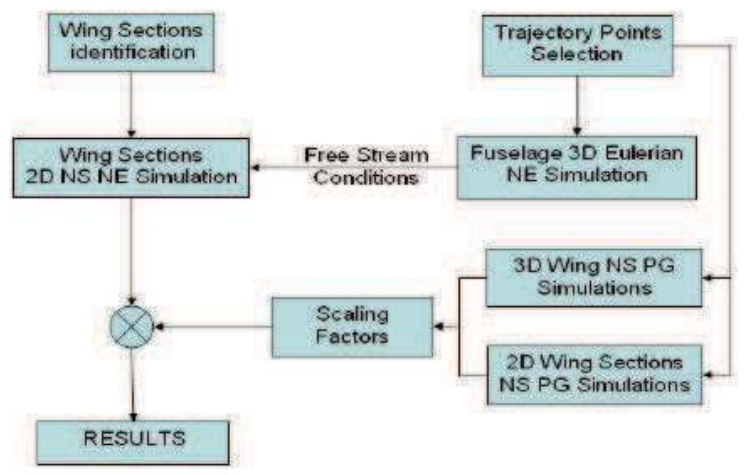

Fig. 8. Flow chart of scaling methodology

The scaling strategy can be summarized as follows: the ratio of the heat flux values of the 2D computation over the $3 \mathrm{D}$ ones, for a certain point along the trajectory, is assumed as scaling factor:

$$
\chi_{s}(x, \bar{y}, t)=\frac{\dot{q}_{3 D}}{\dot{q}_{2 D}}
$$

where $\bar{y}$ indicates a function of the $x$ variable, describing the wing section profile. The scaling factors determined for a subset of selected points along the trajectory have been applied without loss of generality to all the other selected cases, following the equation:

$$
\dot{q}(x, \bar{y}, \bar{t})_{N E}^{3 D}=\chi_{s}(x, \bar{y}, t)_{P G} \cdot \dot{q}(x, \bar{y}, \bar{t})_{N E}^{2 D}
$$


In eq. 2 the scaling factor $\chi_{s}$ has been evaluated by applying perfect gas hypothesis both to 3D wing-alone and to 2D computations. Moreover it has to be pointed out that the scaling factor has obviously not been determined for each point of the trajectory so suitable interpolation with time has been used. In this way, it has been possibile to have an estimation of heat flux distribution over the wing along the trajectory by reducing the needed CPU time and, anyhow, with a good level of approximation.

An orbital re-entry trajectory obtained with preliminary aerodynamic and inertial databases is available for the FTB-X vehicle. In order to be able to respect a thermal barrier of $1100 \mathrm{~kW} / \mathrm{m}^{2}$ at the nose stagnation point a guidance strategy with a modulation of AoA has been adopted. Due to the relatively high efficiency of the vehicle a very long re-entry time is obtained. In Fig. 9 the profiles of Altitude, Mach number and AoA are reported versus Time elapsed, starting from an altitude of $120 \mathrm{~km}$. The descending phase is performed with an angle of attack exceeding 20Âr deg in order to reach a sufficiently low energetic level and low speeds. More in detail, ten different points along the re-entry trajectory have been considered for the CFD analysis according to the described methodology.

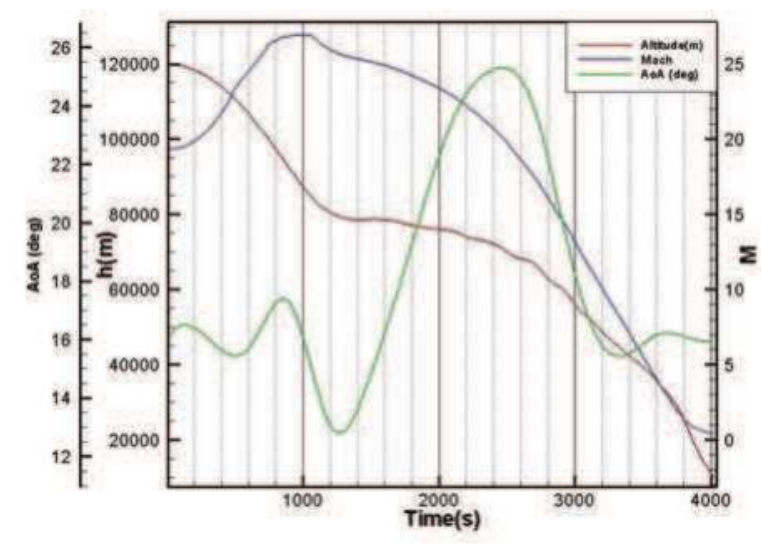

Fig. 9. Reference trajectory

The effects of the bow shock generated by the nose some Eulerian, thermo-chemical non equilibrium computations have been performed over the fuselage geometry. In order to understand the level of approximation related to this assumption a comparison with a full vehicle Navier-Stokes non equilibrium computation has been carried out and the results are reported in Fig. 10 for one point of the trajectory. It is evident that the error in the position of the bow shock is not very significant.

Once determined the freestream conditions for each profile, two-dimensional planar steady state $^{1}$ non equilibrium computations have been performed for the selected points along the flight trajectory in order to obtain a time history of heat flux spatial distribution. From the 3D Eulerian computations, it comes out that the section 1 ( $y=0.550 \mathrm{~m}$ from the symmetry plane of the vehicle) and section $2(y=0.760 \mathrm{~m})$ are positioned in a part of the wing that is inside the bow shock for all the selected points along the flight trajectory, whereas section 3 ( $y=0.915 \mathrm{~m})$ is outside the bow shock at higher altitudes therefore it feels an undisturbed flow field, and

\footnotetext{
${ }^{1}$ Characteristic flow time are of order of milliseconds for this regimes therefore the assumption of steady state with respect to the variation of freestream conditions along the flight trajectory is fully adequate.
} 


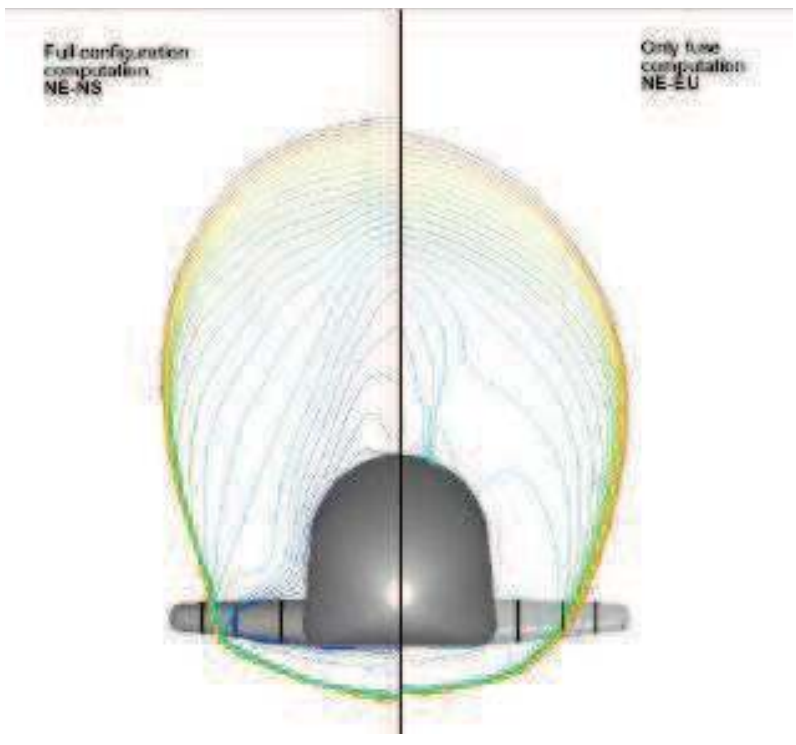

Fig. 10. $M \infty=20.29$ AoA=25.30 deg: Mach Contours

it is inside the bow shock for the lower altitudes points because the shock angle increases as Mach number decreases.

In Fig. 11 the stagnation point heat flux over the selected profiles is reported versus the flight time. All the computations have been performed in laminar conditions with radiative equilibrium assumption at the wall; clearly, according to the $2 \mathrm{D}$ computations, the profile 2 $(y=0.760 \mathrm{~m})$ experiences the highest heat flux along the trajectory, with a maximum equal to about $2.1 \mathrm{MW} / \mathrm{m}^{2}$ reached at point 5 of the trajectory $(\mathrm{h}=75 \mathrm{~km}, \mathrm{M} \infty=22.79)$. Of course it has to be kept in mind that these results refer to the 2D computations and that they do not take into account the effect due to the finite span length of the wing therefore in order to predict realistic value of heat flux over the wing, it is necessary to scale these results to take into account the three-dimensionality of the flow, as sweep angle effect and finiteness of the wing. Three-dimensional computations have been performed assuming a perfect gas environment, for some of the selected points along the trajectory to suitably describe the plateau of heat flux during the re-entry trajectory, considering the undisturbed freestream variables as inlet conditions. For each of the selected points, additional two-dimensional computations, respect to those already presented, have been performed assuming a perfect gas environment and undisturbed freestream conditions for all the sections; these additional computations are necessary to find out the scaling factors are reported in Eq. 1. In Fig. 12 pressure coefficient and heat flux distribution over the wing is shown for one of the the trajectory points mentioned above.

In order to provide the heat flux distribution over the wing along the trajectory, two-dimensional non equilibrium computations have been then scaled according to the scaling factors obtained through three-dimensional computations around the wing (see Eq. 2). The results of the scaling methodology are reported in Fig. 13: in particular, in Fig. 13(a) it is reported the variation along the trajectory of the stagnation point heat flux over the three selected profiles whereas in Fig. 13(b) it is reported the heat flux distribution over the three selected profiles for one point of the trajectory. Maximum heat flux is almost constant in 


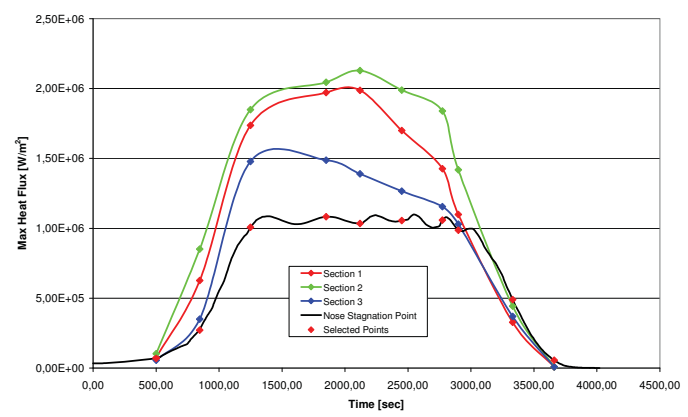

Fig. 11. Two-dimensional stagnation point heat flux over the selected profiles

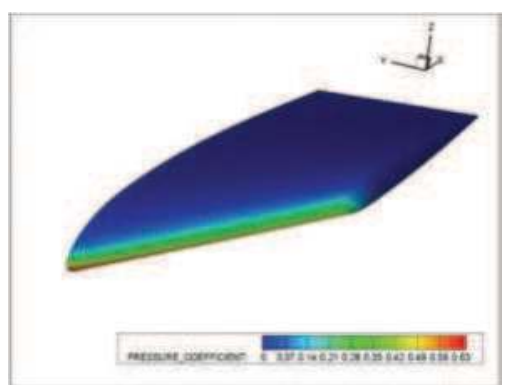

(a) Pressure coefficient

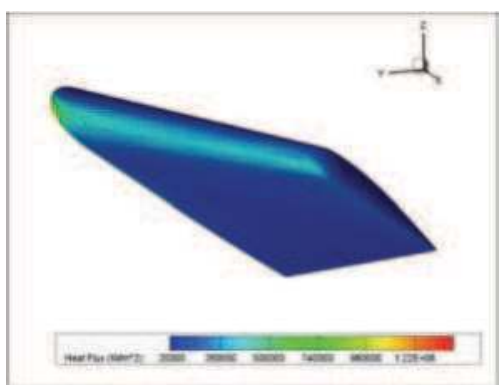

(b) Heat flux

Fig. 12. Results over the wing

the middle part of the trajectory for the section 1 and 3 and equal, respectively, to about 400 $\mathrm{kW} / \mathrm{m}^{2}$ and $300 \mathrm{~kW} / \mathrm{m}^{2}$, whereas it increases for the section 2 up to a value slightly above $400 \mathrm{~kW} / \mathrm{m}^{2}$ reached after $2000 \mathrm{~s}$. The stagnation point is located immediately downstream of the leading edge (due to the effect of the angle of attack), then, after the rapid expansion, it assumes an almost constant value along the entire geometry. The difference on the heat flux value between leeside and windside is lower than $100 \mathrm{~kW} / \mathrm{m}^{2}$.

\section{PWT test design}

Hereinafter a brief description of the activities carried out to numerically design the PWT test are reported. The starting point for the design of a test to be performed in the Plasma Wind Tunnel Scirocco, is the definition of the requirements, generally in terms of heat flux and pressure to be realized on the model, that is necessary to achieve during the test itself. For the considered test, the requirements were determined through the simplified procedure already described; loads foreseen during the re-entry trajectory of FTB-X vehicle are summarized, for the different part of the model, in Table 1 in terms of heat flux. These loads have been used to design the TPS therefore they have to be reproduced in the wind tunnel in order to validate the design procedure. For each part of the model in the same table it 


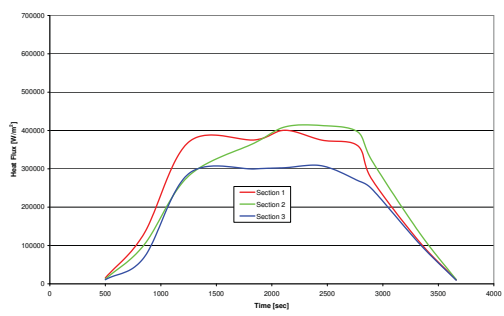

(a) Time variation

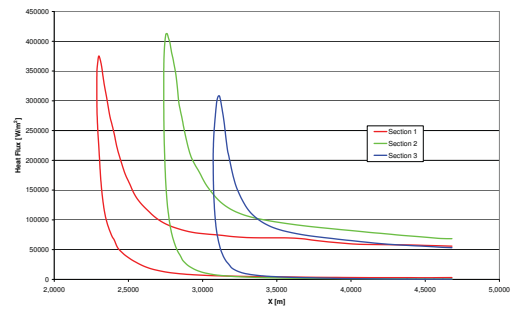

(b) Spatial distribution

Fig. 13. Heat flux over the selected profiles

\begin{tabular}{|c|c|c|c|c|c|}
\hline \multirow[t]{2}{*}{ ID req. } & \multirow[t]{2}{*}{ Test Article Part } & \multicolumn{2}{|c|}{ Wall Conditions } & \multirow[t]{2}{*}{$\mathrm{q}\left[\mathrm{kW} / \mathrm{m}^{2}\right]$} & \multirow[t]{2}{*}{ Position } \\
\hline & & Catalysis & Temp & & \\
\hline A & LE UHTC & FRC & Rad.Eq. & 410 & Stagnation Point \\
\hline B & WINDSIDE PANEL & FRC & Rad.Eq. & 170 & Panel Apex $-x=0.2 m$ \\
\hline $\mathrm{C}$ & LE AC & FCW & Tfix & 410 & Stagnation Point \\
\hline $\mathrm{D}$ & LEESIDE PANEL & FCW & Rad.Eq. & 90 & Panel Apex $-\mathrm{x}=0.1 \mathrm{~m}$ \\
\hline
\end{tabular}

Table 1. Requirements for PWT test design

is shown also the wall boundary conditions, in terms of catalysis and temperature, to be considered. The test has been numerically designed, in terms of values of reservoir enthalpy and pressure and model positioning/attitude within the test chamber to be considered, through an extrapolation-from-flight methodology already used for previous test campaigns as reported in Marini et al. (2007).

For what concerns the present test, the requirement was to achieve a value of heat flux over the MMC panel equal to $90 \mathrm{~kW} / \mathrm{m}^{2}$ and the following operating conditions and model attitude have been determined:

- Reservoir Enthalpy = $13.5 \mathrm{MJ} / \mathrm{kg}$.

- Reservoir Pressure $=3.6$ bar.

- Final Model attitude $=35 \mathrm{deg}$ ( $25 \mathrm{deg}$ mechanical angle $+10 \mathrm{deg}$ of MSS tilting).

- Distance from nozzle exit: $950 \mathrm{~mm}$.

In order to guarantee the safety of the test both in terms of flow blockage occurrence and test article integrity it was decided to consider some intermediate steps for the test procedure. These steps can be summarized as follow:

- insertion of the model with a mechanical angle of attack of 25 deg at a distance from the nozzle exit section equal to $200 \mathrm{~mm}$;

- backward movement of the test article up to a distance from the nozzle exit section equal to $950 \mathrm{~mm}$;

- $10 \mathrm{deg}$ tilting to reach the final attitude of $35 \mathrm{deg}$ that assures the achievement of the requirements over the model. 
The achievements of the right operating conditions within the test chamber during the test is assured by the presence of a copper water-cooled hemi-spherical calibration probe which is inserted in the plasma flow before the insertion of the model to measure the stagnation point heat flux and pressure. Facility regulation parameters (air mass flow and current in the arc) are tuned in order to match on this probe a certain couple $\left(P_{s}, q_{s}\right)$ which corresponds to the desired operating condition in terms of the couple $\left(H_{0}, P_{0}\right)$ evaluated through the extrapolation-from-flight methodology generally used. For the present test, the requirement was to achieve on the calibration probe a stagnation heat flux equal to $870 \mathrm{~kW} / \mathrm{m}^{2}$ and a stagnation pressure equal to 10 mbar. The application of such procedure requires to carry out a lot of CFD computations of the flowfield around the model. Therefore, in order to reduce the CPU time, the flow has been simulated considering a two-dimensional approach. The validity of this hypothesis has been verified through a comparison between two and three-dimensional results. In Fig. 14 heat flux distribution over the surface of the test article is shown; the represented configuration refers to the model equipped with the actively cooled leading edge. Heat flux distribution on the leading edge is strongly bi-dimensional for a great portion of the span. In Fig. 15 the comparison between two and three-dimensional flowfield on the symmetry plane is shown; close to the leading edge the position and the shape of the bow shock are practically the same whereas a certain difference has been found downstream where the stronger expansion due to the three dimensional side effects leads a deviation of the shock toward the wall.

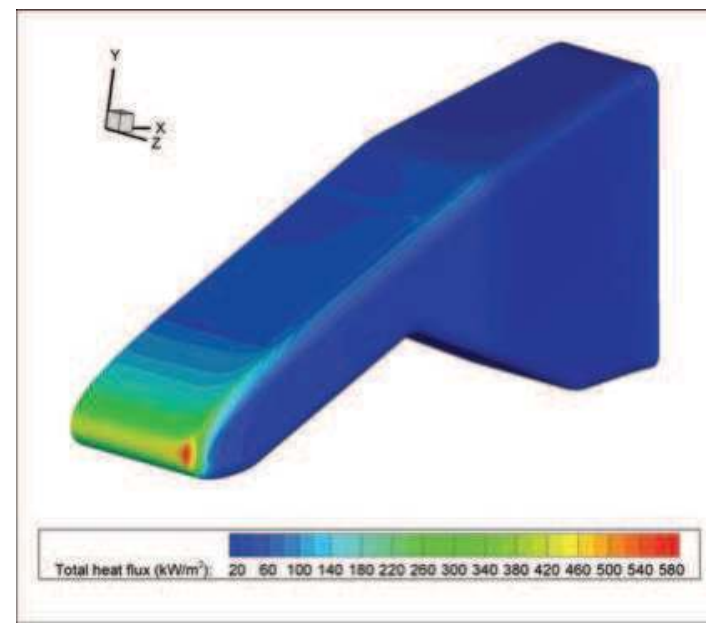

Fig. 14. Heat flux distribution over the test article

\subsection{Model flowfield simulation}

As already said, in order to guarantee the safety of the test, either in terms of flow blockage either in terms of structural integrity of the model, the adopted test procedure foreseen some intermediate steps before the final test condition. For each of the selected conditions, in terms of model positioning and angle of attack a numerical computation has been performed as summarized in Table 2. The four computations have been performed assuming a steady state of the flow by neglecting possible non-stationary effects due to the movement of the model within the test chamber. Wall temperature has been considered equal to $300 \mathrm{~K}$ for all the parts of the model in the first phases of the test in order to provide conservative results for heat flux 


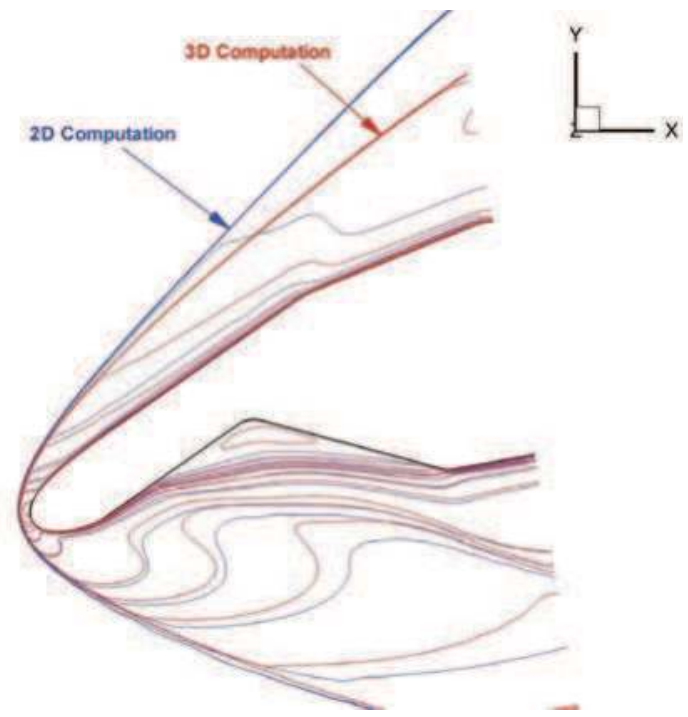

Fig. 15. Comparison between 2D and 3D flowfield on the symmetry plane

Wall Temperature

\begin{tabular}{|c|c|c|c|c|c|}
\hline Run ID & Description & Position & AoA & LE WS & LS \\
\hline 1 & Insertion of the model & $x=200 \mathrm{~mm}$ & 25 & 300300 & 300 \\
\hline 2 & Backward movement & $\mathrm{x}=950 \mathrm{~mm}$ & 25 & 300300 & 300 \\
\hline 3 & Tilting & $x=950 \mathrm{~mm}$ & 35 & 300300 & 300 \\
\hline 4 & End of the test & $\mathrm{x}=950 \mathrm{~mm}$ & 35 & $410 \mathrm{RE}$ & RE \\
\hline
\end{tabular}

Table 2. CFD Matrix

estimation, whereas it has been assumed equal to the radiative equilibrium temperature (with different emission coefficients) for the $4^{\text {th }}$ computation, except than for the AC leading edge which has been considered at a fixed wall temperature equal to $410 \mathrm{~K}$. For what concerns the catalytic conditions, the leading edge and MMC panel have been considered as fully catalytic, whereas the $\mathrm{C}-\mathrm{C} / \mathrm{SiC}$ panel has been considered as partially catalytic. Heat flux distribution computed in Run-4 over the model is shown in Fig. 16(a); peaks, clearly visible at the interfaces between the different part of the model, are due to discontinuities in the boundary conditions in terms of catalytic behaviour and emission coefficients. In Fig. 16(b), the detail of the heat flux on the MMC panel for the four computations of Table 2 is shown: at model insertion (Run-1) the maximum heat flux reaches a value slightly above $100 \mathrm{~kW} / \mathrm{m}^{2}$ either for the effect of wall temperature (equal to $300 \mathrm{~K}$ ) either the reduced distance from nozzle exit section; the backward movement (Run-2) causes a decrease of heat flux over the model because the expansion of the flow continues also in the test chamber outside the conical nozzle; the tilting of the MSS (Run-3) leads an increase of heat flux, then in Run-4 a decrease of heat flux (till the final value at the tip equal to $90 \mathrm{~kW} / \mathrm{m}^{2}$, as for the requirement) is found due to the effect of the radiative equilibrium hypothesis. 


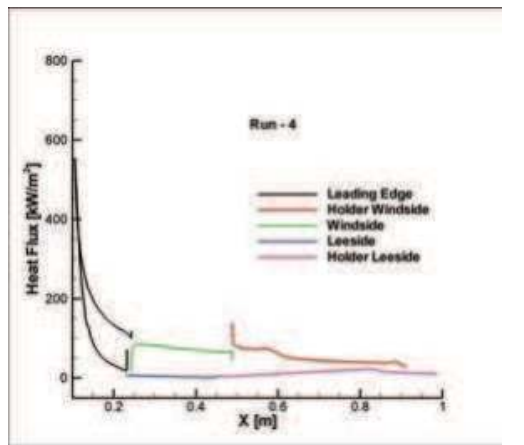

(a) run-4

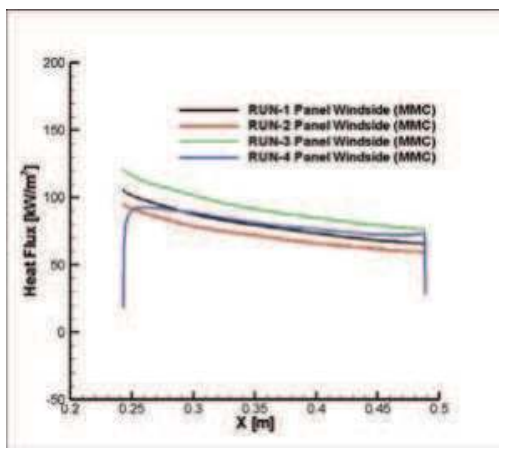

(b) detail of the tip

Fig. 16. Heat flux over the model

\section{Test execution}

The test has been executed on October 2008; in Fig. 17 the model within the test chamber, ready to test, is shown. On the left side it is visible the expansion nozzle exit section with 1950 $\mathrm{mm}$ of diameter whereas on the right the diffuser need to slow down the hypersonic flow and for the recovery of pressure is visible. During the test, after the ignition of the arch heater, the qualification of the flow within the test chamber has been done through the hemispherical calibration probe; air mass flow and current have been then tuned to match the requirements. Nominal values of heat flux and pressure measured at the probe stagnation point during the test were slightly different with respect to those determined before the test due to the necessity to maintain stable conditions in the arc heater but, in any case, within the associated measurement uncertainty; in detail, heat flux was equal to $930 \mathrm{~kW} / \mathrm{m}^{2}$ with a difference with respect to the required value of $60 \mathrm{~kW} / \mathrm{m}^{2}$, whereas wall pressure was 11 mbar instead of 10 mbar.

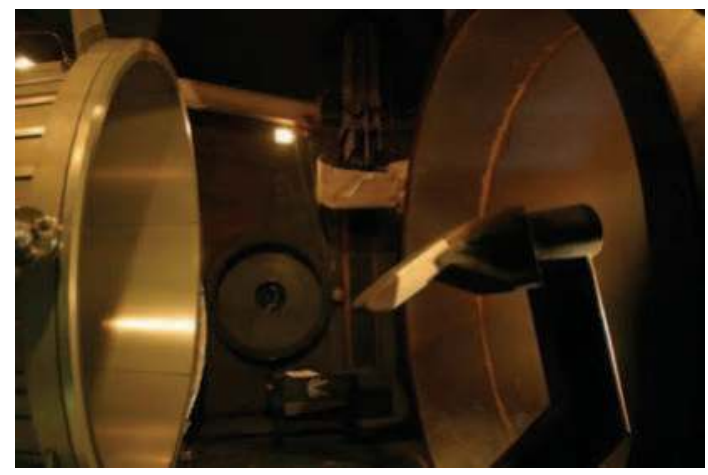

Fig. 17. Model ready to be tested

In Fig. 18 different images during the test execution are shown and they are specified also in the following:

- (a). model enter the plasma flow;

- (b). model arrives at centreline at a distance from nozzle exit section of $200 \mathrm{~mm}$; 
- (c). after 570s the backward movement starts;

- (d). model reaches the final position, distance from nozzle exit section $950 \mathrm{~mm}$;

- (e). after 10s in this position the model rotates to achieve the final angle of attack of 35 deg;

- (f). after $300 \mathrm{~s}$ in this position the test finishes.

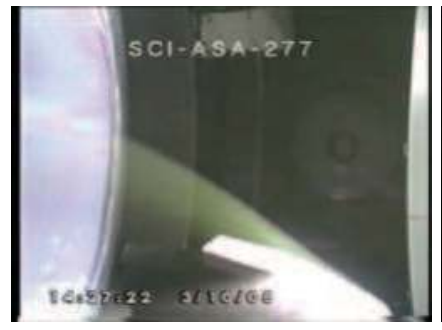

(a)

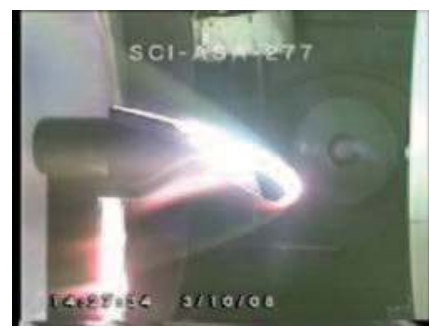

(d)

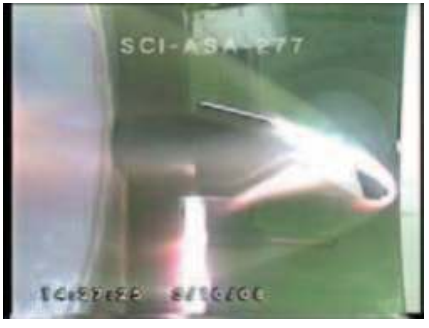

(b)

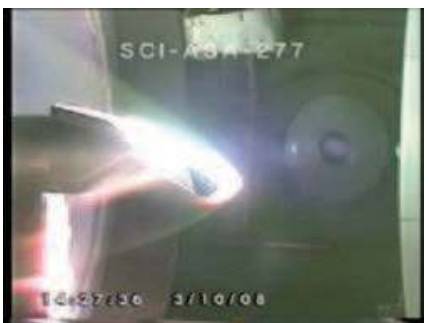

(e)

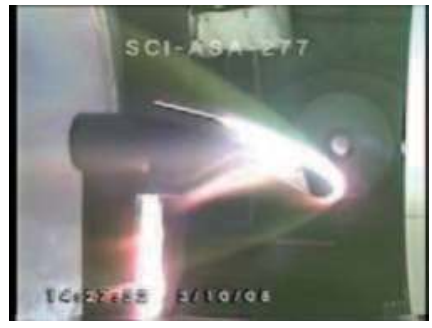

(c)

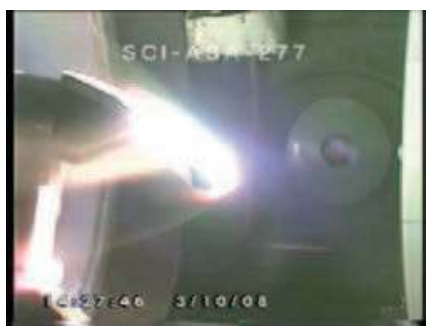

(f)

Fig. 18. Test execution

The test total test duration was 916s starting from the insertion of the model in the plasma flow; the numerical rebuilding, whose results will be reported hereinafter, has been done considering the first part before the backward movement and the rotation of the model.

\section{IR measurements}

Not intrusive thermographic techniques for temperature measurement have always played an important role among the diagnostics of the wind tunnel facilities. It has been developed and it is foreseen to become even more important for the future. During the present test, surface temperature measurements have been carried out with IR thermography to collect useful data for post-test analysis and comparison with numerical prediction tools (see also Di Clemente et al. (2008)). A proper calibration, using a blackbody Mikron M300, has been performed on the temperature range expected during the test; this apparatus is a compact portable blackbody calibration source with a built-in digital indicating controller that can be set at any temperature between ambient $5^{\circ} \mathrm{C}$ and $1150^{\circ} \mathrm{C}$. Once set, the source temperature is controlled to within $\pm 0.2^{\circ} \mathrm{C}$ by an internal RTD sensor. The unit has an emissivity of 0.966 in the 8-12 $\mu$ wavelength range (the range of acquisition of the thermocamera AGEMA Thermovision 900). The M300 is easy to transport to a calibration location, either in laboratory or in the plant. It has a dedicated PID controller with $0.1^{\circ} \mathrm{C}$ resolution.

The thermocamera has been positioned on the top of the test chamber to acquire measurements on the upper part of the model; the positioning has been made by optimizing 
the view angle in the different phases of the test as injection in the plasma flow, backward movement and tilting. The total time of acquisition has been equal to $45 \mathrm{~min}$, starting from the injection in the plasma flow $(t=0 \mathrm{~s})$, considering the first $15 \mathrm{~min}$ of the test itself and 30 min of the cooling phase after the shut down of the facility. In Fig. 19 some images of the thermocamera in different time instants are reported.

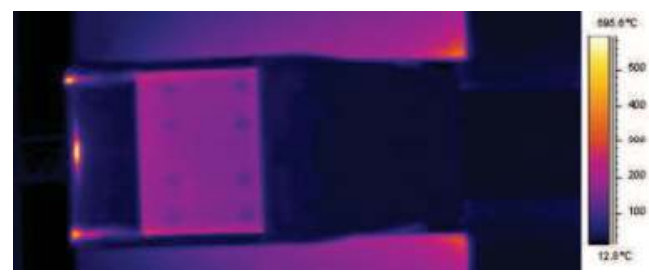

(a) $\mathrm{t}=10 \mathrm{~s}$

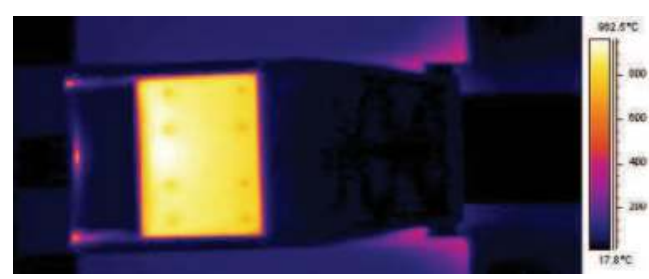

(c) $\mathrm{t}=420 \mathrm{~s}$

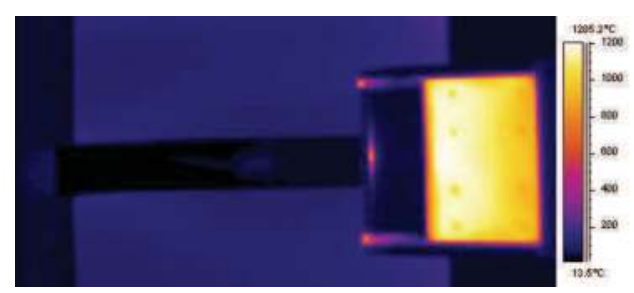

(e) $\mathrm{t}=900 \mathrm{~s}$

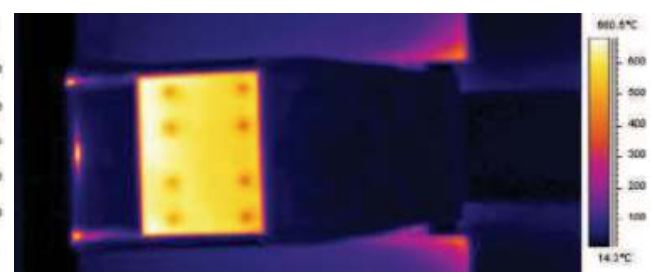

(b) $\mathrm{t}=180 \mathrm{~s}$

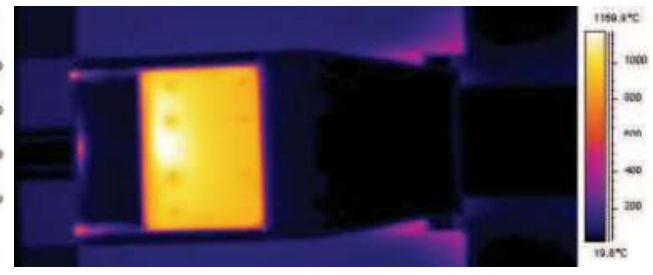

(d) $\mathrm{t}=540 \mathrm{~s}$

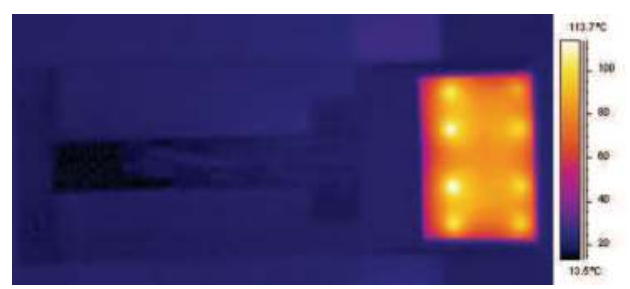

(f) $t=2700 \mathrm{~s}$

Fig. 19. Thermocamera acquisitions

Once the thermocamera is calibrated it is possible to perform all the operations with its infrared acquisition. In order to evaluate the temperature of an object by means of a single thermocamera acquisition, it is necessary to know the value of emissivity of its surface. In the present case, this determination has been made through the comparison of thermocamera acquisition with the measurements provided by a dual color pyrometer pointed on the panel surface. The comparison is shown in Fig. 20 for a value of emissivity equal to 0.91 . The comparison has been made in the second phase of the test, because the pyrometer has been positioned to acquire measurements after the backward movement and for temperatures higher than $800^{\circ} \mathrm{C}$.

In order to provide quantitative and not only qualitative measurements the results of the thermocamera have been extracted along longitudinal and transversal lines over the model shown in Fig. 21; in particular seven lines have been extracted in the longitudinal direction (named from L01 to L07) and four in the transversal one (named from L08 to L11). As an example, in Fig. 22 the results at $\mathrm{t}=180 \mathrm{~s}$ along the longitudinal and transversal lines: at panel 


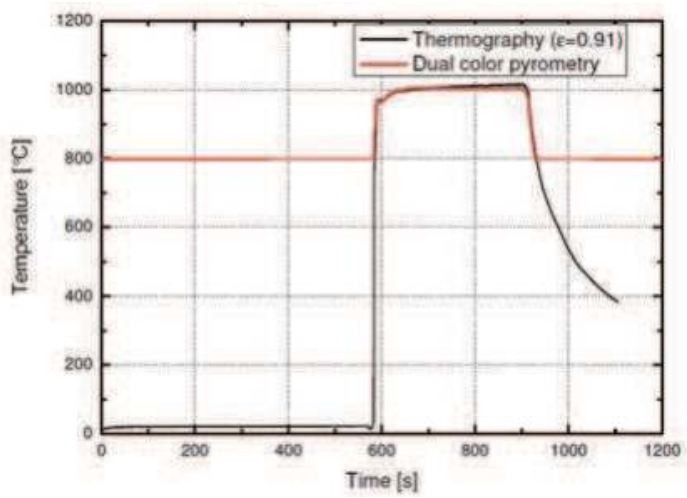

Fig. 20. Comparison between thermocamera and pyrometer acquisition

tip, temperature is low due to the presence of the actively cooled leading edge whose effects extends for $4-5 \mathrm{~cm}$ on the panel (see Fig.22(a); the same is predicted at the trailing edge, due to the model holder that is cooled as well. Transversal lines reported in Fig.22(b) show an almost flat distribution in the central part of the panel and a lateral decreases due to the rounded edges of the model. The presence of the bolts which fix the panel to the underlying structure causes a local decrease of temperature due to the enhanced heat conduction. In Fig.23, time evolution of temperature along the simmetry line and one of the transversal lines is reported: temperature increases quickly in the first $180 \mathrm{~s}$ of the test then it seems to reach an almost constant condition even though it continues slowly to increase. In order to better analyze time dependant behaviour of temperature, the evolution versus time in two points of the panel, located on the simmetry line near the tip (P5) and near the trailing edge (P12) is shown in Fig.24:

- the initial heating is almost quick, temperature reaches a value of about $800^{\circ} \mathrm{C}$ at point $\mathrm{P5}$ and $600^{\circ} \mathrm{C}$ at point $\mathrm{P} 12$ in about $200 \mathrm{~s}$;

- after this time, the slope of the curve decreases and temperature continues to increase slowly reaching an almost constant behaviour;

- at $\mathrm{t}=570$ s the model is moved downstream and then rotated of $10 \mathrm{deg}$ to reach an angle of attack respect to the flow equal to $35 \mathrm{deg}$ (this part of the acquisition is neglected to the high error associated to the measurement);

- after the rotation temperature starts to increase again due to the higher value of heat flux up to the shut down of the facility after $900 \mathrm{~s}$;

- the last phase of the acquisition regards the cooling of the model.

\section{Numerical rebuilding and comparison with experimental results}

Generally, an aerothermodynamic simulation to estimate heat flux is carried out considering the wall as an isothermal surface or adiabatic, with or without the hypothesis of radiative equilibrium; computed heat flux values are then used to simulate the thermal field inside the material. As matter of fact, due to the intrinsic unsteady behaviour of the test under investigation and to the thermal properties of the materials involved this uncoupled approach could be not adequately accurate for the rebuilding of the test itself; therefore, it has been 


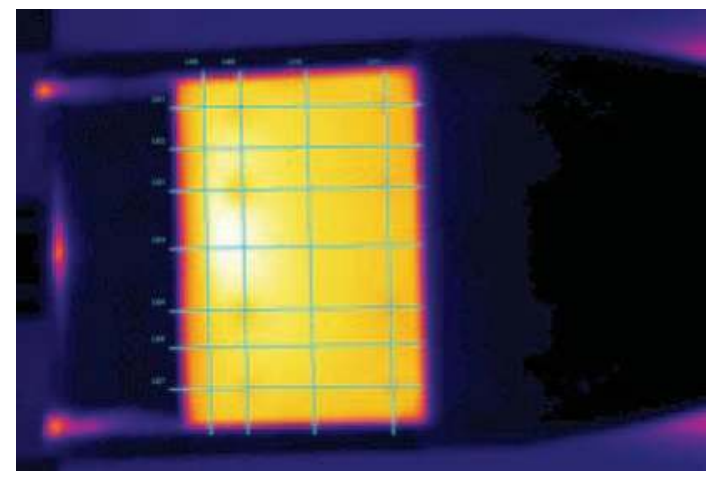

Fig. 21. Extraction lines for thermocamera results

carried out considering a coupled simulation between the aerodynamic and the internal thermal field.

\subsection{Set point definition}

As already specified, the achievement of the desired test conditions within the test chamber is assured by the insertion in the plasma flow of the hemispherical calibration probe. Numerical rebuilding, consequently, has to be carried out considering the actual values of heat flux and pressure measured by the probe. In this case, considering these values, the operating condition in terms of reservoir enthalpy and pressure which allows to obtain these results on the probe have been determined and then used to compute the flow inside the PWT conical nozzle and then around the model for the rebuilding of the test. This procedure assures a good characterization of the flow within the test chamber and then that the selected operating condition is suitable for the rebuilding of the results over the model. The selected condition for the rebuilding is characterized by a reservoir enthalpy of $13.7 \mathrm{MJ} / \mathrm{kg}$ and a reservoir pressure equal to 3.9 bar.

\subsection{Aerothermal coupling}

The numerical rebuilding procedure has been applied considering a weak coupling between the external fluidynamic field and the internal thermal field, considering different freestream conditions ahead the model according to its movement within the test chamber. By analyzing the characteristic times of the phenomenon, it has been deduced that it was possible to consider different steady state computations of the fluidynamic field whose dynamic behaviour is much faster than heat propagation inside the material, on the other side, through unsteady thermal simulations. In synthesis, the internal thermal field in the panel has been evaluated through a series of successive unsteady computations considering the heat flux due to the external flow as a fixed input for each step; at the end of each thermal computation, this input due to the external flow has been updated by repeating the CFD computations considering either different freestream conditions due to the movement of the model within the test chamber either the updated wall temperature distribution derived from the thermal analysis. Due to the simmetry of the flow and to the already shown "almost" two dimensional characteristics of the flowfield around the model, numerical rebuilding has been made considering two-dimensional planar computations therefore the results will be compared with those obtained on the simmetry line through the thermocamera system. Moreover only the upper part of the model has been simulated. The rebuilding has been done starting from the 


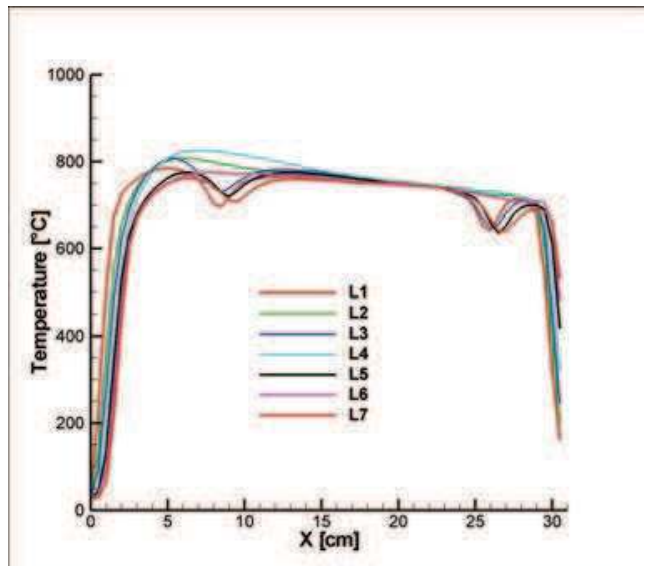

(a) simmetry line

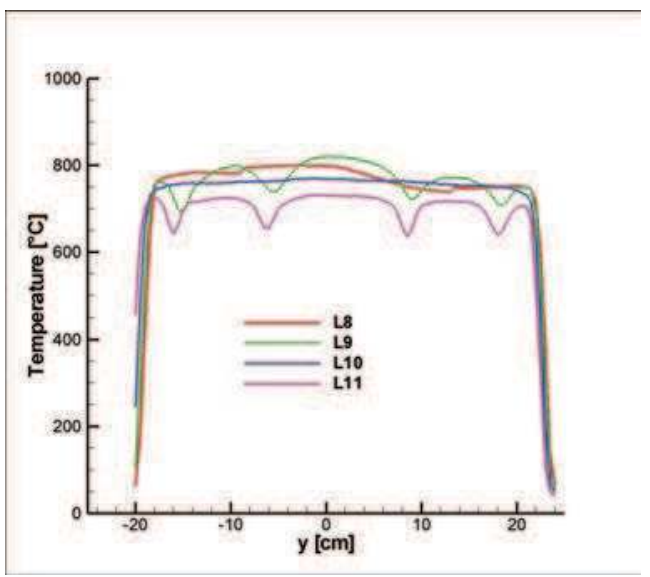

(b) transversal line (L10)

Fig. 22. Temperature acquisition at $\mathrm{t}=180 \mathrm{~s}$

entrance of the model in the plasma flow by carrying out 9 computations (CFD + thermal) as shown in Table 3 where for each time also the positioning of the model, in terms of distance from nozzle exit and angle of attack, is indicated. After each CFD computation, the thermal state inside the panel is computed through an instationary simulation of a certain time, and at the end, wall temperature distribution is imposed as boundary condition for the following CFD computation. Moreover, during the instationary thermal simulation, the effect of temperature on heat flux at the wall is accounted for through the following equation:

$$
q_{\text {wall }}(x, T)=h_{c}(x, \bar{T}) \cdot\left(h_{\text {aw }}-c p \cdot T_{\text {wall }}\right)
$$

being $h_{c}(x, \bar{T})$ evaluated at the beginning of each thermal simulation from the results of the previous CFD calculation. 


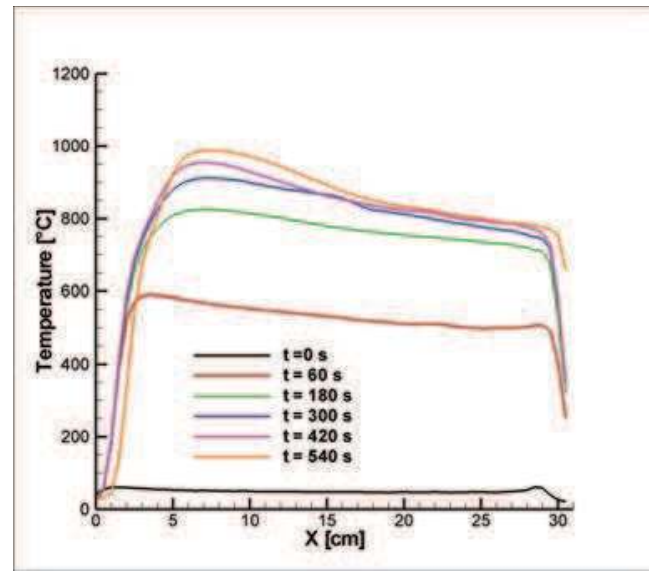

(a) simmetry line

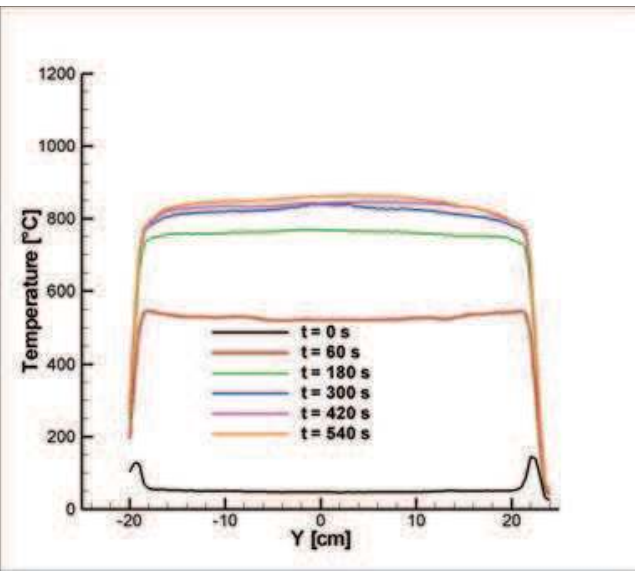

(b) transversal line

Fig. 23. Temperature time history

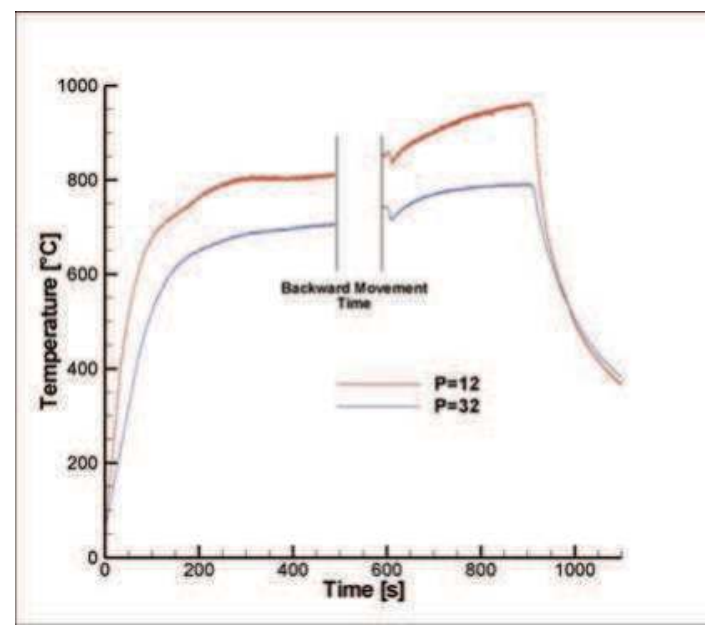

Fig. 24. Temperature time evolution

In Fig. 25 external and internal temperature field at the end of the first phase (i.e. $t=570 \mathrm{~s}$ ) is shown; heat flux propagation inside the panel, whose temperature reaches values of about $800 \mathrm{~K}$, is quite evident whereas the underlying layer, which protect the inner part of the model, remains relatively cold due to its insulating properties.

Temperature evolution over the surface for this first phase is reported in Fig.26 and compared with the experimental results extracted from the thermocamera measurements. The comparison shows a rather good agreement; the experimental results are affected by the cooling of the leading edge and the holder that is not simulated in the numerical rebuilding therefore the comparison at the tip and the trailing edge of the panel is not meaningful. In order to compare the time evolution of temperature on the panel, two points located near the tip and near the trailing edge have been considered. The results are shown in Fig.27. The 


\begin{tabular}{|c|c|c|c|}
\hline Run II & $\begin{array}{c}\text { Time } \\
\text { [s] }\end{array}$ & $\begin{array}{l}\text { from n } \\
{[\mathrm{mm}]}\end{array}$ & $\begin{array}{l}\text { AoA } \\
\text { [deg] }\end{array}$ \\
\hline 1 & 0 & 200 & 25 \\
\hline 2 & 570 & 200 & 25 \\
\hline 3 & 575 & 450 & 25 \\
\hline 4 & 580 & 750 & 25 \\
\hline 5 & 589 & 950 & 25 \\
\hline 6 & 597 & 950 & 25 \\
\hline 7 & 603 & 950 & 30 \\
\hline 8 & 612 & 950 & 35 \\
\hline 9 & 916 & 950 & 35 \\
\hline
\end{tabular}

Table 3. Conditions for the numerical rebuilding

comparison between numerical and experimental results is quite good for the first phase of the test, both during the initial heating (from $t=0 \mathrm{~s}$ to $t=200 \mathrm{~s}$ ) and the following phase up to the steady state (from $t=200 \mathrm{~s}$ to $\mathrm{t}=570 \mathrm{~s}$ ). On the other side, after the backward movement, the comparison shows some discrepancies. In this case, in addition to the error associated to the numerical simulation there is also the increased uncertainty associated to the experimental measurement induced by a non optimized angle of view between the thermocamera and the model since this factor was optimized for the first position of the model. The behaviour of the curve is represented quite well by the numerical results, being differences found in the absolute value of temperature of about $50 \mathrm{~K}$. The same consideration apply also for the analysis of the cooling phase.

\section{Conclusions}

The experimental and numerical activities carried out for the analysis of the PWT test on the wing leading edge model have been presented. The IR thermo-graphic images have been processed through an ad-hoc developed mapping procedure to be directly compared with the results of the numerical analysis carried out through a non standard approach based on the unsteady simulation of the test and a coupling between the external aerodynamic field and the internal thermal field. Differences of about $100 \mathrm{~K}$ have been found between the experimental and numerical results at the end of the test. It has been found that these differences increased during the second phase of the test when the angle of view between the thermocamera and the panel was not optimized. It has to be remarked that some uncertainties can be also associated to this type of numerical rebuilding especially for what concerns the surface characteristics of the material, in terms of emissivity (for thermocamera measurement) and catalytic behaviour (for CFD numerical rebuilding). In order to use experimental data to validate the numerical models, the main sources of these uncertainties should be reduced. In detail:

- It should be preferable to have direct measurements of the heat flux which can be directly compared with the numerical results.

- The real test conditions should be determined more in detail; a variation of the energetic level in the plasma flow during the test can cause a different dissociation of air mixture ahead the model and therefore different catalytic recombination processes at the wall. The measurement of the heat flux carried out in the test chamber through the calorimetric copper probe (fully catalytic), beyond the instrumental uncertainties, is not able to completely describe the different energetic contributes of the flow exiting from the 


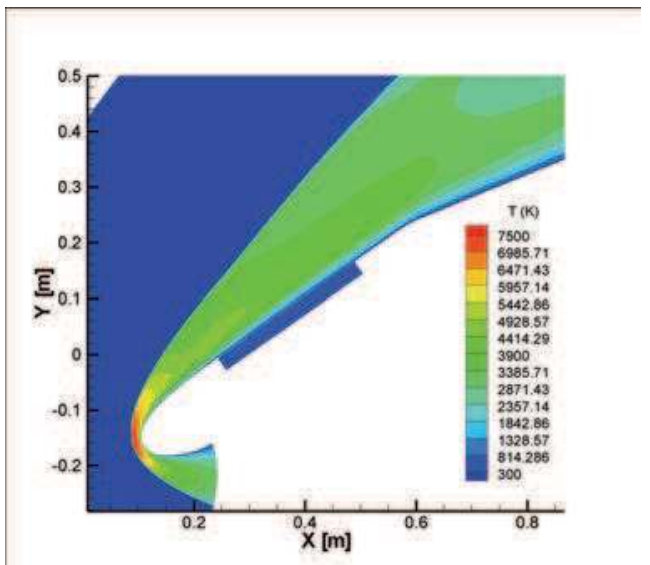

(a) entire field

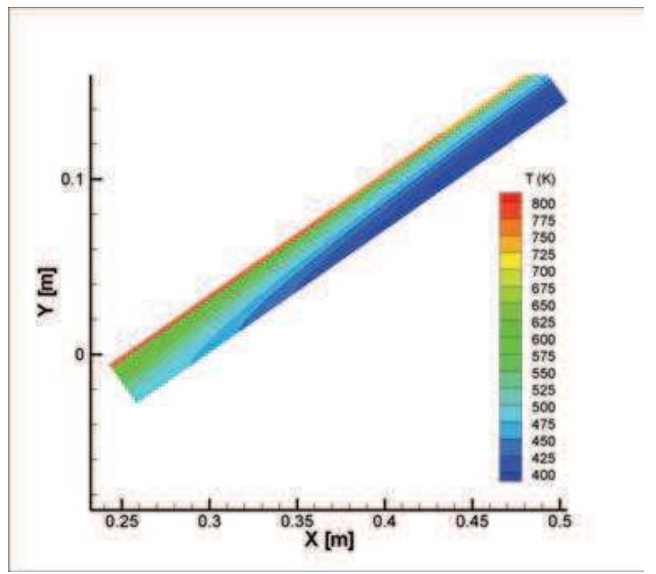

(b) panel

Fig. 25. External and internal temperature field at $\mathrm{t}=570 \mathrm{~s}$

expansion nozzle. As matter of fact, the measured heat flux is the sum of the convective and diffusive term whereas the correct repartition of these two terms is important especially dealing with partially catalytic materials.

- The uncertainties related to the knowledge of the material should be reduced: not only in terms of emissivity or catalysis but also thermal conductivity, capacity and density to accurately rebuild also the thermal field inside the model.

In any case, the possibility to apply the developed numerical/experimental procedure to rebuild a so articulated test, due to the adopted procedure and the dimensions of the model itself, has been demonstrated even though the main sources of errors, both from an experimental and numerical point of view, need to be further reduced. 


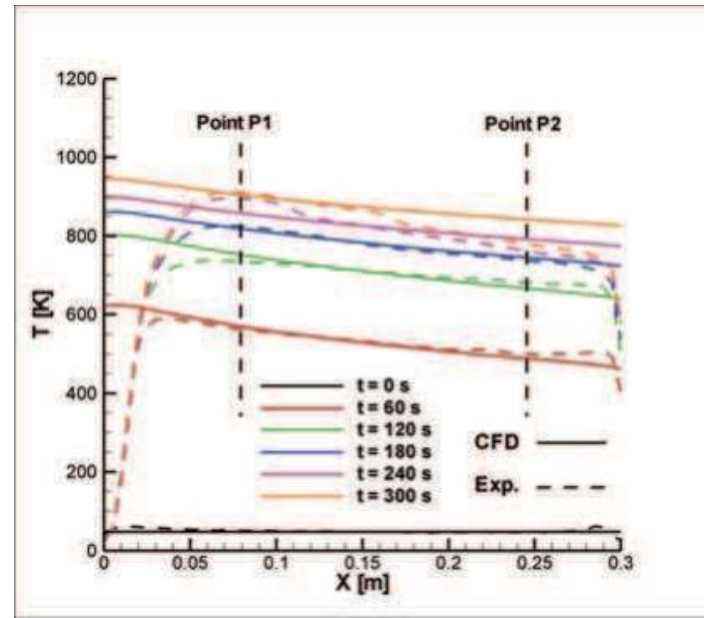

Fig. 26. Temperature evolution for different time istants

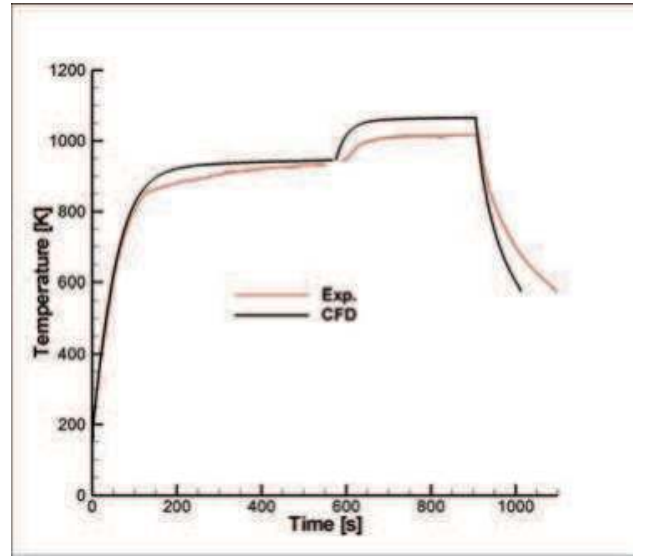

(a) point P1

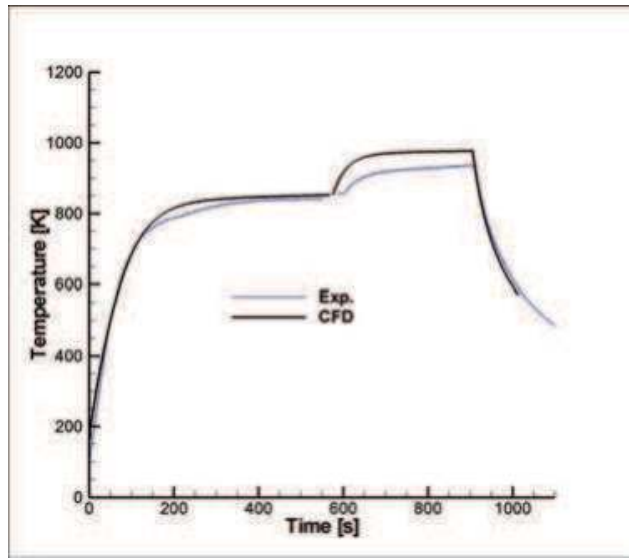

(b) point P2

Fig. 27. Time evolution of temperature on the panel

\section{Acknowledgments}

Authors would like to thank the Italian Space Agency, that funded the current project, in particular dr. Emanuela D'Aversa, and Thales Alenia Space as Prime contractor and test article assembly responsible, in particular the Program Manager dr. Roberto Viotto and Project Manager, dr. Franco Fossati. They are also grateful to the CIRA project manager dr. Giuliano Marino and to the PWT facility team, in particular to Eduardo Trifoni for his work during the entire project. 


\section{References}

Battista, F., Rufolo, G. \& Di Clemente, M. (2007). Aerothermal environment definition for a reusable experimental re-entry vehicle wing, Proceedings of $39^{\text {th }}$ AIAA Thermophysics Conference. Miami, USA.

Borrelli, S. \& Pandolfi, M. (1990). An upwind formulation for the numerical prediction of non equilibrium hypersonic flows, $12^{\text {th }}$ International Conference on Numerical Methods in Fluid Dynamics. Oxford, United Kingdom.

De Filippis, F., Caristia, S., Del Vecchio, A. \& Purpura, C. (2003). The scirocco pwt facility calibration activities, Proceedings of $3^{r} d$ International Symposium Atmospheric Reentry Vehicle and Systems. Arcachon,France.

Di Clemente, M., Rufolo, G., Cardone, G. \& Ianiro, A. (2008). Aerothermal coupling methodology for the rebuilding of a plasma wind tunnel test and comparison with an advanced infrared measurement technique, Proceedings of $6^{t} h$ European Symposium on Aerothermodynamics for Space Vehicles. Versailles, France.

Edney, B. (1968). Anomalous heat transfer and pressure distribution on blunt bodies at hypersonic speeds in the presence of an impinging shock wave, Technical report, FFA. Rep.115.

Marini, M., Di Benedetto, S., Rufolo, G., Di Clemente, M. \& Borrelli, S. (2007). Test design methodologies for flight relevant plasma wind tunnel experiments, Proceedings of West-East High Speed Flow Field Conference, Moscow,Russia.

Pezzella, G., Battista, F., Schettino, A., Marini, M. \& De Matteis, P. (2007). Hypersonic aerothermal environment preliminary definition of the cira $\mathrm{ftb}-\mathrm{x}$ reentry vehicle, Proceedings of West-East High Speed Flow Field Conference. Moscow, Russi.

Ratti, F., Muylaert, J., Gavira, J. \& Walpot, L. (2008). European experimental re-entry testbed expert: Qualification of payloads for flight, Proceedings of $6^{t} h$ European Symposium on Aerothermodynamics for Space Vehicles. Versailles,France.

Tumino, G. (2006). Hypersonic systems and technologies developments at esa, $14^{\text {th }}$ AIAA/AHI International Space Planes and Hypersonic Systems and Technologies Conference. Camberra, Australia. 


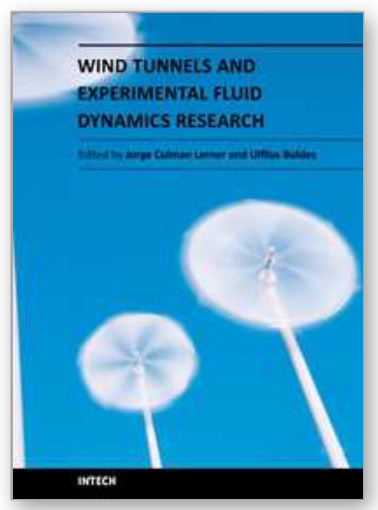

\author{
Wind Tunnels and Experimental Fluid Dynamics Research \\ Edited by Prof. Jorge Colman Lerner
}

ISBN 978-953-307-623-2

Hard cover, 709 pages

Publisher InTech

Published online 27, July, 2011

Published in print edition July, 2011

The book â€œWind Tunnels and Experimental Fluid Dynamics Researchâ€ is comprised of 33 chapters divided in five sections. The first 12 chapters discuss wind tunnel facilities and experiments in incompressible flow, while the next seven chapters deal with building dynamics, flow control and fluid mechanics. Third section of the book is dedicated to chapters discussing aerodynamic field measurements and real full scale analysis (chapters 20-22). Chapters in the last two sections deal with turbulent structure analysis (chapters 23-25) and wind tunnels in compressible flow (chapters 26-33). Contributions from a large number of international experts make this publication a highly valuable resource in wind tunnels and fluid dynamics field of research.

\title{
How to reference
}

In order to correctly reference this scholarly work, feel free to copy and paste the following:

Giuseppe C. Rufolo, Francesco Battista, Adolfo Martucci and Marco Di Clemente (2011). Design, Execution and Rebuilding of a Plasma Wind Tunnel Test compared with an Advanced Infrared Measurement Technique, Wind Tunnels and Experimental Fluid Dynamics Research, Prof. Jorge Colman Lerner (Ed.), ISBN: 978-953307-623-2, InTech, Available from: http://www.intechopen.com/books/wind-tunnels-and-experimental-fluiddynamics-research/design-execution-and-rebuilding-of-a-plasma-wind-tunnel-test-compared-with-anadvanced-infrared-meas

\section{INTECH}

open science | open minds

\author{
InTech Europe \\ University Campus STeP Ri \\ Slavka Krautzeka 83/A \\ 51000 Rijeka, Croatia \\ Phone: +385 (51) 770447 \\ Fax: +385 (51) 686166 \\ www.intechopen.com
}

\author{
InTech China \\ Unit 405, Office Block, Hotel Equatorial Shanghai \\ No.65, Yan An Road (West), Shanghai, 200040, China \\ 中国上海市延安西路65号上海国际贵都大饭店办公楼 405 单元 \\ Phone: +86-21-62489820 \\ Fax: +86-21-62489821
}


(C) 2011 The Author(s). Licensee IntechOpen. This chapter is distributed under the terms of the Creative Commons Attribution-NonCommercialShareAlike-3.0 License, which permits use, distribution and reproduction for non-commercial purposes, provided the original is properly cited and derivative works building on this content are distributed under the same license. 